\title{
la dysplasie ectodermique anhidrotique : à propos de deux cas
}

RÉSUMÉ Les dysplasies ectodermiques constituent un groupe hétérogène de maladies qui touchent les organes d'origine ectodermique ainsi que leurs dérivés. Dans cet article, les auteurs rapportent les cas de deux sœurs atteintes d'une forme anhidrotique de la maladie ou syndrome de Christ

\section{Bassima CHAMI}

Professeur assistante

en odontologie chirurgicale,

Faculté de médecine dentaire,

Rabat, Maroc.

\section{El Miloud RAHMANI}

Professeur agrégé

en prothèse adjointe,

Hôpital Militaire d'Instruction

Mohamed V (H.M.I.M.V.) Rabat,

B.P. 6363 Madinate Al Irfane Rabat.

Naima NAOUMI

Orthodontiste,

H.M.I.M.V., Rabat.

Abderrahmane HAFID

Maître assistant en ODF,

H.M.I.M.V., Rabat.

Mohamed EL HOURI

Professeur agrégé en dermatologie,

H.M.I.M.V., Rabat.

Bouabid EL MOHTARIM

Professeur de l'enseignement supérieur en prothèse adjointe,

Chef du service d'odontologie,

H.M.I.M.V., Rabat.

Wafae EL WADY

Chef de service

d'odontologie chirurgicale

C.C.T.D., Rabat.
Siemens Touraine. Les manifestations dentaires et leur traitement sont particulièrement soulignés.
MOTS CLÉS

hypohidrose

hypotrichose

hypodontie

prothèse dentaire 


\section{introduction}

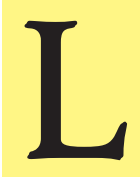

a dysplasie ectodermique anhidrotique ou Syndrome de Christ-Siemens-Touraine est une génodermatose rare liée au chromosome X. Sa prévalence est de $1 / 100.000$ naissances $[1,8,16,18]$.

C'est une histodysplasie congénitale et familiale atteignant surtout la peau et ses annexes. Les deux sexes peuvent être atteints avec une prédominance masculine en rapport avec le mode de transmission le plus souvent récessif. Ce syndrome est caractérisé par :

- une hypohidrose ;

- une hypotrichose ;

- une hypodontie ou anodontie[7, 15].
Les accidents hyperthermiques des premiers mois de la vie représentent le risque majeur de cette affection et peuvent entraîner la mort ou des séquelles neuropsychiques[2, 7,17$]$.

Le chirurgien-dentiste a un rôle très important dans le diagnostic et la prise en charge bucco-dentaire, le but étant un développement harmonieux des structures maxillo-faciales.

À travers deux cas familiaux (deux sœurs), les auteurs soulignent le retentissement de cette maladie sur le développement des structures maxillofaciales et le rôle du chirurgien-dentiste dans le rétablissement des fonctions altérées.

\section{observations}

\section{- observation $\mathrm{n}^{0} 1$}

Mlle F.K., âgée de 19 ans, en bon état de santé général, consulte pour absence de dents et tassement de l'étage inférieur, motifs aussi bien esthétique que fonctionnel. L'examen exobuccal montre un front bombé, des oreilles légèrement décollées, une exophtalmie, une diminution de la dimension verticale d'occlusion et une protrusion des lèvres. L'examen endobuccal révèle :

- des dents atypiques : $11,15,16,17$, $21,23,25,33$ et 35 ;

- la persistance de certaines dents lactéales abrasées : $71,72,81$ et 82 .

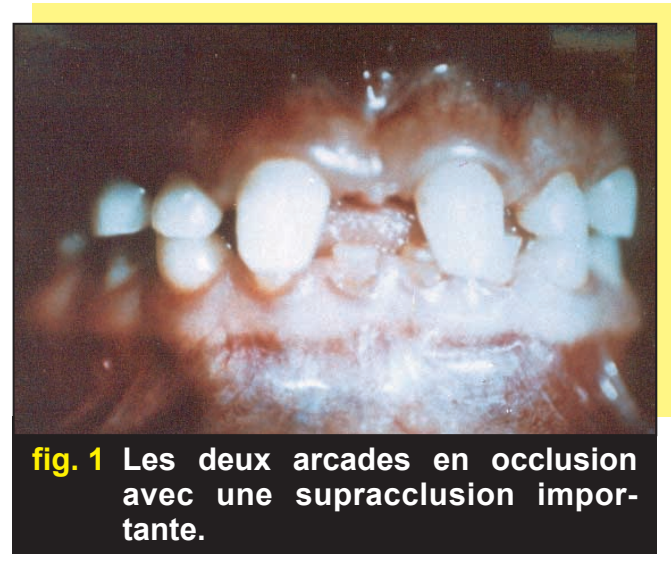

- une supraclusion importante (fig. 1). Le bilan radiologique montre : les agénésies des $12,14,18,22,24,28,38$, $37,34,32,31,41,42$ et 44 (fig. 2). 


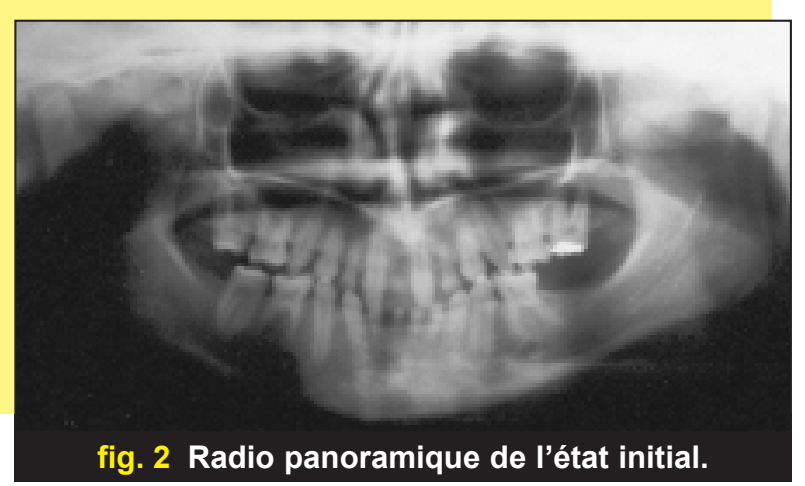

Le schéma dentaire résiduel est :

\begin{tabular}{lllllll|lllllll}
17 & 16 & 15 &. & 13 & 52 & 11 & 21 & 62 & 23 & $\cdot$ & 25 & 26 & 27 \\
\hline 47 & 46 & 45 & $\cdot$ & 43 & 82 & 81 & 71 & 72 & 33 & $\cdot$ & 35 & 36 &.
\end{tabular}

À ces anomalies s'ajoutent des troubles - des cils et sourcils raréfiés ; cutanéo-phanériens caractérisé par :

- des ongles fragiles et cassants.

- une atrophie cutanée ;

Ces éléments nous ont permis de poser

- une kératodermie et une hyperhydrose palmo-plantaire ; le diagnostic de Dysplasie Ectodermique Anhidrotique (DEA) confirmé par nos

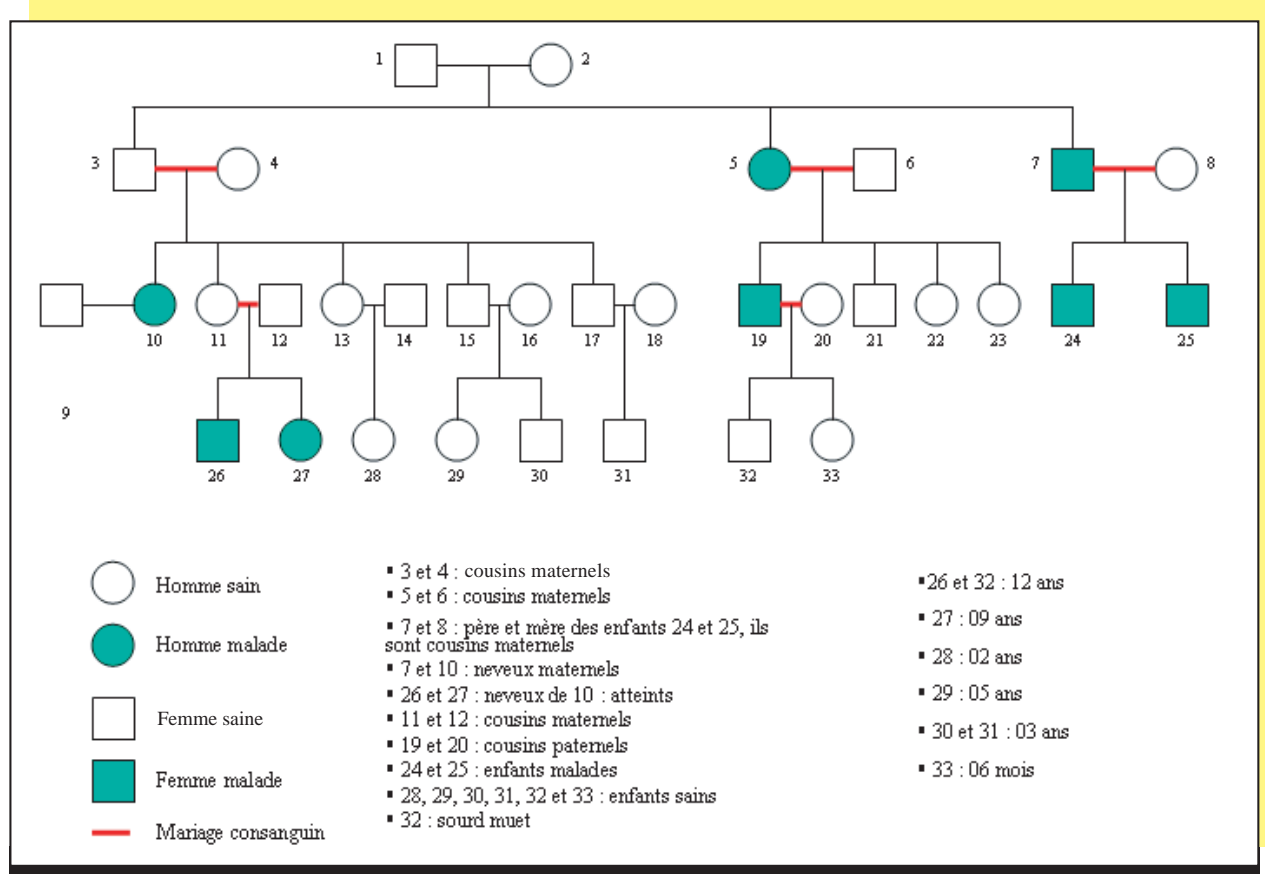

fig. 3 Arbre généalogique illustrant la transmission de la DEA sur quatre générations. 


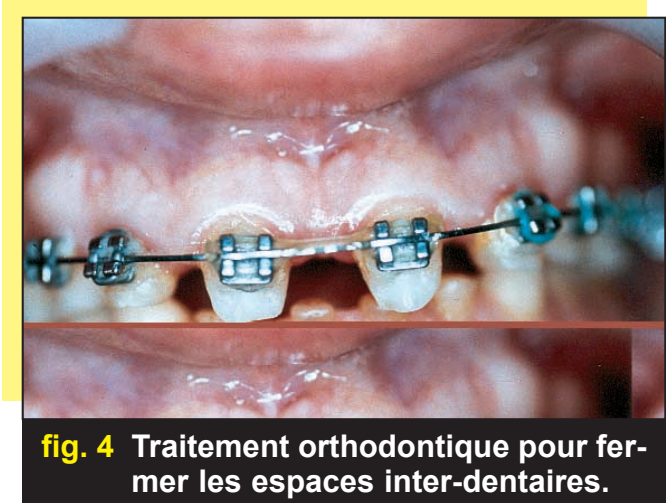

collègues dermatologues. L'enquête familiale nous a permis de révéler des cas similaires (fig. $\mathbf{3}$ ).

Le traitement a visé le rétablissement $d u$ capital dentaire ce qui n'élimine pas l'objectif esthétique. Pour ce faire, nous avons réalisé, dans un premier temps, un traitement orthodontique (fig. 4) suivi d'un traitement prothétique qui a consisté :

- au maxillaire, en la réalisation d'un bridge antérieur après extraction de la 52 et 62 ;

- à la mandibule, en la réalisation d'une prothèse adjointe partielle immédiate après extraction des dents lactéales (fig. 5).

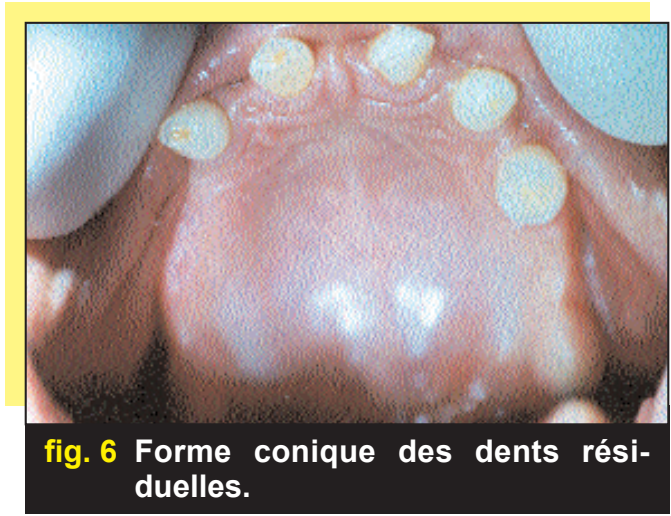

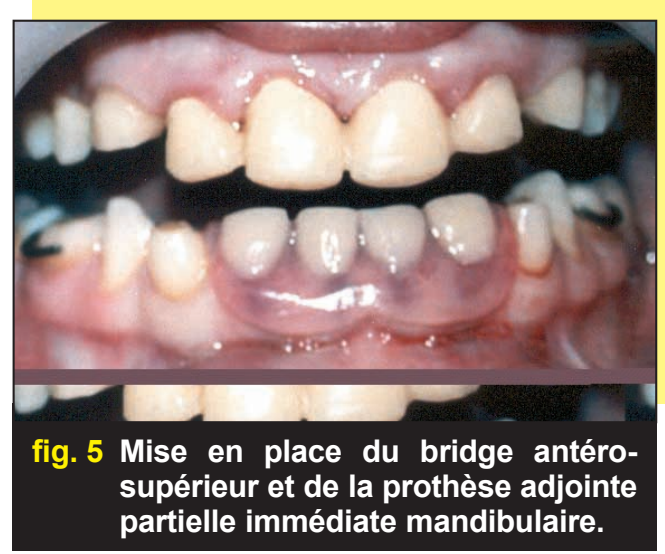

- observation $\mathrm{n}^{\circ} 2$

Mlle F.S., âgée de 17 ans consulte en même temps et pour les mêmes raisons que sa sœur aînée. L'interrogatoire révèle un retard mental.

L'examen exobuccal fait apparaître :

- des oreilles décollées ;

- un front bombé ;

- le nez ensellé ;

- une diminution de la dimension verticale d'occlusion.

L'examen endobuccal révèle :

- un articulé inversé ;

- une prognathie mandibulaire ;

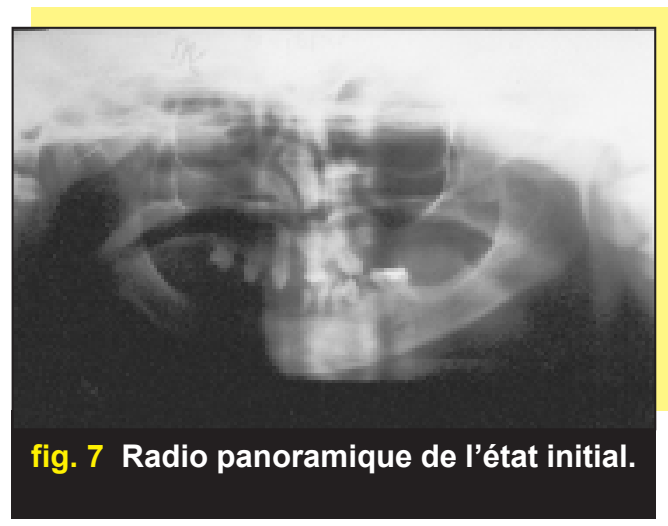


- la persistance de dents lactéales de forme atypique (conique) : 53, 63 et 61 (fig. 6).

L'examen radiologique montre (fig. 7 ) :

- les agénésies de : 12, 13, 14, 15, 16,
$17,18,21,22,23,24,25,26,27,28$, $38,37,35,34,33,32,31,41,42,43$, $44,45,46,47$ et 48 ;

- la résorption des racines des dents lactéales résiduelles.

- Le schéma dentaire est :

\begin{tabular}{cccccc|cccccc}
. & 55 &. & 53 &. & 11 & 61 &. & 63 &. & 65 &. \\
\hline 64 & $\cdot$ & 84 & 83 & 82 & 81 & 71 &. &. &. &. & 36
\end{tabular}

L'examen cutanéo-phanérien révèle :

- une peau fine, sèche et lisse surtout au niveau des mains ;

- une kératodermie et une hyperhidrose palmo-plantaire ;

- des cheveux secs, légèrement décolorés avec des zones d'alopécie diffuse ;

- des cils et sourcils raréfiés et clairsemés avec alopécie partielle (queue du sourcil) ;
- des ongles de forme normale mais fragiles et cassants.

Pour répondre aux objectifs esthétiques et fonctionnels de cette patiente nous avons procédé, dans un premier temps, à l'extraction des dents mobiles et celles présentant une résorption sévère, puis nous avons réalisé des prothèses subtotales au maxillaire pour passer enfin à des prothèses totales bimaxillaires (fig. 8, 9 et 10).
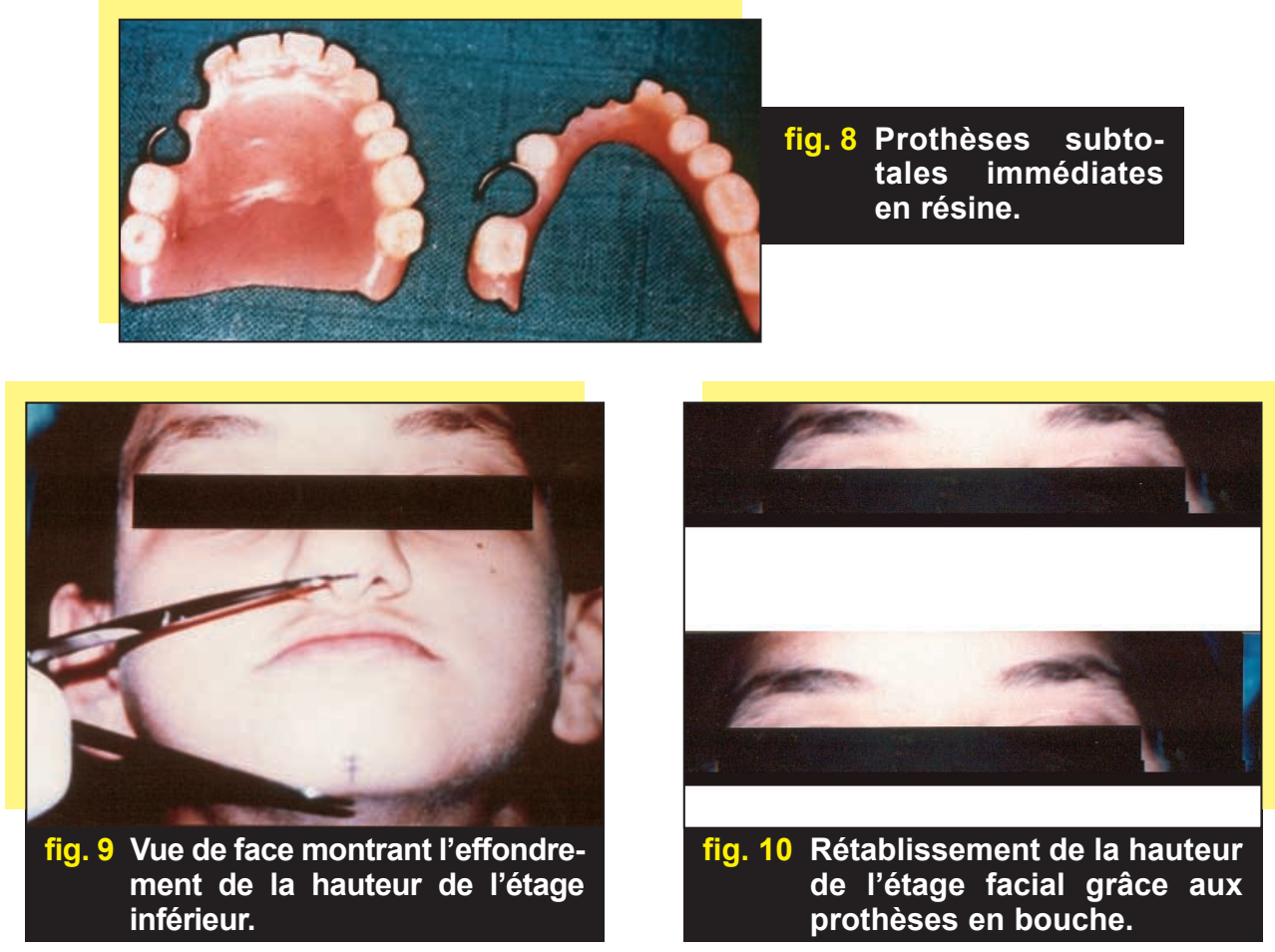


\section{discussion}

L'expression phénotypique de la dysplasie ectodermique anhidrotique est très variable, et le diagnostic peut s'avérer difficile, notamment chez les femmes puisque cette génodermatose se transmet dans la majorité des cas sur un mode récessif lié à l'X[7].

Habituellement, la maladie se manifeste par une triade symptomatique constante associée plus ou moins à d'autres signes qui sont inconstants.

\section{- hypohidrose ou anhidrose}

Elle désigne une diminution voire une abolition de la sécrétion sudorale consécutive à l'aplasie des glandes sudoripares[15]. Elle attire l'attention dès le plus jeune âge, mise en évidence par l'examen stéréomicroscopique du revêtement cutané et par la biopsie cutanée effectuée à la paume des mains ou sur la face antérieure des avantbras. La peau des patients atteints de cette affection est souvent fine, lisse, sèche, soyeuse au toucher avec parfois un aspect eczémateux des joues et des extrémités. L'anhidrose peut entraîner chez le nourrisson des troubles de la thermorégulation se traduisant par des hyperthermies responsables de déshydratation, de convulsions et parfois même de décès $[2,5,7,9,15]$.

\section{- hypotrichose}

Les sujets affectés présentent des cheveux peu abondants par rapport à la normale, clairsemés, secs, souvent blonds et cassants.
Ils sont presque inexistants sur le vertex et la nuque. La calvitie totale est précoce chez les sujets adultes.

Les cils et les sourcils sont le plus souvent absents ou clairsemés. Le reste de la pilosité manque ou est peu abondant. D'autre part, les ongles peuvent être normaux ou présenter une dysplasie de forme et de couleur, déprimés dans leur partie centrale, friables et secs $[2,7,8]$.

\section{- hypodontie ou l'anodontie}

Elles se caractérisent par des agénésies dentaires multiples et non systématisées touchant les deux dentitions. Les formes mineures d'hypodontie, dites aussi oligodonties s'expriment par des agénésies portant sur les dents de fin de série.

Les formes majeures se traduisant par une anodontie totale bimaxillaire sont rares $[4,8,12,13,20]$.

Les dents présentes sur l'arcade ont souvent une forme et une position anormales : elles sont plus petites, ectopiques, espacées, pointues ou coniques dites «dents de cochon» ou «dents de loup» $[1,5,9,10,13]$.

Un taurodontisme est également observé, pouvant atteindre les deux dentitions lactéale et permanente.

\section{- les signes cliniques associés}

\section{LE FACIÈS}

Le faciès est souvent caractéristique et permet d'évoquer le diagnostic au premier coup d'œil[2, 7, 13, 15, 19]. 
Il est de type sénile, dominé par les signes suivants $[2,7,8,9,12,18,19]$ :

- front bombé et rebords orbitaires marqués ;

- grandes lèvres et oreilles décollées ;

- ensellure nasale marquée ;

- absence de sourcils ;

- yeux écartés ;

- fentes palpébrales obliques ;

- menton petit ;

- angle mandibulaire ouvert.

\section{STRUCTURES} BUCCO-DENTAIRES

Les anomalies dentaires de nombre et de forme ont des répercussions fonctionnelles, esthétiques et psychologiques. L'hypodontie conduit à des troubles de l'articulé avec modifications des rapports osseux. En effet, l'analyse céphalométrique montre des troubles $\mathrm{du}$ sens vertical, sagittal et fron$\operatorname{tal}[2,3,4,6]$.

Dans le sens vertical, la dimension verticale d'occlusion est souvent diminuée entraînant une perturbation de la physiologie des muscles masticateurs et une modification $d u$ faciès (vieillard) $[2,3,7,12,18]$.

Dans le sens sagittal, l'absence d'engrènement dentaire favorise le proglissement de la mandibule entraînant une classe III d'Angle.

Dans le sens frontal, des latérodéviations ont été observées. Aussi, on note d'une part l'absence du développement des procès alvéolaires liée à l'absence des dents et d'autre part, l'hypotonie des lèvres et la position basse de la langue qui ne favorisent pas la croissance des bases osseuses.
Ces perturbations altèrent les fonctions de mastication, de phonation et de déglutition entraînant des préjudices esthétiques responsables de troubles psychologiques : sentiment d'infériorité, problèmes relationnels et problèmes matrimoniaux pour les jeunes filles[2, 3, 4, 6, 14].

- complications générales liées au syndrome

\section{ATTEINTES OCULAIRES}

L'affection peut être accompagnée d'aplasie des glandes lacrymales ainsi que d'agénésie des canaux lacrymaux pouvant engendrer une conjonctivite chronique qui peut évoluer vers une kératite.

On peut aussi observer une blépharite par atrophie ou absence des glandes de Meibomius[2, 7].

\section{ATTEINTES RESPIRATOIRES}

L'absence ou l'hypoplasie des glandes séro-muqueuses de l'appareil respiratoire est assez fréquente. Ceci favorise le développement d'infections respiratoires : broncho-pneumopathies à répétition, infections rhinopharyngées et asthme[7, 9, 12].

\section{RETARD INTELLECTUEL ET STATURO-PONDÉRAL}

Un retard intellectuel est parfois signalé. Il pourrait être en relation avec les troubles de phonation, d'audition et $d u$ retard $d u$ développement staturopondéral dans la petite enfance. Ce dernier est le résultat de malnutrition due aux malformations dento-maxillaires $[2,7]$. 


\section{traitements}

La prise en charge des patients atteints de la DEA doit être pluridisciplinaire : médicale et odonto-stomatologique.

\section{- traitement médical}

Le traitement symptomatique vise surtout à conseiller les parents afin d'éviter le risque d'hyperthermie chez le nourrisson et l'enfant en bas âge.

Dans ce sens, le pédiatre a un rôle clé dans la sensibilisation des familles et pourrait proposer un monitorage des nourrissons à l'aide d'une sonde thermique cutanée pendant le sommeil au moins jusqu'à un an. D'autres précautions doivent être prises : pas d'exposition au soleil, bains fréquents en cas de grosses chaleurs, locaux frais et aérés. Pour lutter contre la sécheresse cutanée, des applications d'huile sont conseillées.

Le traitement symptomatique de la rhinite atrophique consistera en des lavages au sérum physiologique associés parfois à une antibiothérapie pour éviter toute surinfection [2, 17].

\section{a traitement odonto-stomatologique}

Sur le plan odonto-stomatologique, la réhabilitation orale doit être entreprise précocement afin d'assurer le développement harmonieux des maxillaires et ainsi pallier les troubles fonctionnels, esthétiques et psychologiques de l'enfant $[1,10,19]$.

La prise en charge thérapeutique varie en fonction de la sévérité de l'affection, elle dépend aussi de l'âge, de la croissance et du développement du système manducateur tout en tenant compte de la coopération et de la motivation du patient et/ou des parents.

Pendant longtemps, le traitement s'est résumé à l'extraction des dents existantes sur l'arcade et à la confection d'une prothèse totale. Actuellement, la tendance est à la conservation des organes présents car ils maintiennent l'os alvéolaire, la sensibilité proprioceptive et constituent des éléments de rétention des prothèses $[2,7]$.

\section{TRAITEMENT ORTHODONTIQUE}

Le traitement orthodontique est souvent réalisé en phase pré-prothétique afin d'aligner les dents, corriger leurs axes, optimiser leurs rapports, ouvrir ou fermer des diastèmes (fig. 4) ou encore pour tracter une dent incluse $[2,6,7,10]$.

\section{LA RÉHABILITATION PROTHÉTIQUE}

Le traitement prothétique doit être le plus précoce possible[4,14] afin de :

- restaurer les fonctions de mastication et de phonation ;

- améliorer la déglutition ;

- rétablir une dimension verticale adéquate ;

- favoriser une croissance faciale normale.

En absence des dents, les prothèses totales sont les plus utilisées. La réalisation de ces prothèses impose quelques impératifs spécifiques, notam- 
ment, l'établissement d'une relation de confiance avec le jeune patient et les parents permettant d'assurer le suivi et d'adapter la prothèse en fonction de la croissance [8, 11, 18].

Les différentes étapes de conception sont identiques à celles des prothèses conventionnelles pour adultes avec parfois quelques difficultés dues au manque de stabilité et de rétention secondaire à l'atrophie des bases osseuses et à la sécheresse buccale accompagnant le syndrome $[4,14,18]$.

Certains auteurs proposent de disposer d'un vérin transversal sur la prothèse dont l'activation va permettre d'accompagner la croissance.

Des rebasages réguliers de la prothèse, voire même sa réfection, permettent d'adapter celle-ci à la croissance. La présence de certaines dents sur l'arcade permet d'opter pour la réalisation d'over dentures qui présentent l'avantage de préserver l'os alvéolaire et la sensibilité proprioceptive et de rétablir la dimension verticale d'occlusion $[2,6,8,11]$.

La prothèse fixe est peu indiquée du fait du caractère provisoire des restaurations.

La pose d'implants chez l'enfant est un élément controversé du fait de la croissance. Elle a fait l'objet d'une conférence de consensus qui a permis la pose d'implants dans le cas d'anodontie réalisant ainsi des prothèses implanto-portées.

Ces implants seront placés de préférence vers l'âge de 5 à 6 ans dans la zone antérieure mandibulaire[4, 8, 11, 16].

\section{conclusion}

La dysplasie ectodermique anhydrotique reste un syndrome encore mal connu. Le risque de mortalité élevé durant les premiers mois de vie de l'enfant, ainsi que toutes les complications respiratoires consécutives à une hyperthermie par hypohidrose rendent nécessaire le dépistage précoce de la maladie ainsi que le conseil génétique qui doit être proposé aux femmes conductrices.
Le chirurgien-dentiste joue un rôle important, bien que tardif dans le dépistage des formes frustes du syndrome en mettant en évidence les anomalies dentaires se rapportant à la maladie. Il joue aussi un rôle important dans la réhabilitation maxillofaciale de ces patients et ce, dès leur jeune âge.

\section{bibliographie}

1. Artis JP, et al. Conséquences dentaires et maxillo-faciales de la dysplasie ectodermique anidrotique.
Actual Odonstomatol 1992;180:773-789.

2. Abadi B, Herren C. Clinical treatment of ectodermal dysplasia: a case report.

Quintessence Int 2001 32:743-745.

3. Boj JR, et al. Cephalometric changes 
in patient with ectodermal dysplasia after placement of dentures. J Clin Pediatr Dent 1993; 17(4):217-220.

4. Bonin B, et al. Traitement implantaire précoce d'un enfant atteint de dysplasie ectodermique anhidrotique : à propos d'un cas. Rev Stomatol Chir Maxillofac 2001;102(6):313-318.

5. Buchon $\mathrm{R}$, et al. Le syndrome de Christ-Siemens-Touraine : signes radiologiques et cliniques.

Revue Imagerie Médicale 1992;4:835-837.

6. Cetiner $D$, et al. Clinical management of ectodermal dysplasia with long term follow up: two cases report. $\mathrm{J}$ Clin Pediatr Dent 2001;25(3):187-190.

7. Cozlin A, et al. La dysplasie ectodermique anhidrotique ou syndrome de Christ-Siemens-Touraine : à propos d'un cas de forme légère. Med Bucc Chir Bucc 2000; 6(1);21-26.

8. Esteban $D$, et al. Over denture prothesis for oral rehabilitation of hypohidrotic ectodermal dysplasia: A case report. Quintessence Int 1997; 28(10):657-663.

9. Fraysse, et al.

Le syndrome de

Christ-Siemens-Touraine : incidences thérapeutiques. Rev Stomatol Chir Maxillofac 1987;88(3):185-189.

10. Itt-Hagarun $A$, et al. Oral rehabilitation of hypohidrotic ectodermal dysplasia patient: A 6 year follow-up. Pediatr Dent 2000;31(9): 642-648.

11. Jimenz-Castellanos $E$, et al. Anodontie associée à une dysplasie ectodermique anhidrotique : à propos d'un cas clinique. Rev Odonto Stomatol 2000; 29(1):17-22.

12. Masse JF, Perusse R. Ectodermal dysplasia. $\mathrm{J} \mathrm{Br}$ Pediatr Assoc 1994; 71(2):1-2.

13. Moreau JL, Terrisse JP. Un cas de dysplasie ectodermique anhidrotique chez trois frères : problèmes odontologiques. Actual Odontostomatol 1983;143:469-477.

14. Naser B.

La prothèse adjointe totale et subtotale chez les enfants atteints de dysplasie ectodermique (anodontie, oligodontie).
Rev Odonto Stomatol 1997; 26(2-3):123-131.

15. Pavlov $\mathrm{ML}$, et al. Étude d'un cas de dysplasie ectodermique $X$ dépendante. Actual Odontol 1998;203: 367-375.

16. Smith RA, et al. Placement of an endosseous implant in a growing child with ectodermal dysplasia. Oral Surg Oral Med Oral Pathol 1993;75:669-673.

17. Testard $\mathrm{H}$, et al. Dysplasie ectodermique anhidrotique. Arch Pediatr 1991;48:343-345.

18. Ramos $V$, et al. Complete dentures for a child with hypohidrotic ectodermal dysplasia: A clinical report.

J Prosthet Dent 1995:329-331.

19. Vierucci $S$, et al. Dental and craniofacial findings in hypohidrotic ectodermal dysplasia during the primary dentition phase. J Clin Pediatr Dent 1994; 18(4):291-297.

20. Yedomon $\mathrm{HG}$, et al. Dysplasie ectodermique : à propos de six cas familiaux chez des enfants de race noire. Ann Dermatol Venereol 1994;121:123-126.

\section{SUMMARY}

\section{Anhidrotic ectodermal dysplasia: two cases report}

B. CHAMI, E. M. RAHMANI, N. NAOUMI, A. HAFID, M. EL HOURI, B. EL MOHTARIM, W. EL WADY

Ectodermal dysplasia is an heterogeneous group of diseases that affect numbers of ectodermal origin organs as well as their derivatives. We present two cases of sisters who caught an anhidro- tic ectodermal displasia or Christ Siemens Touraine syndrome. Dental manifestations of the disease and therapeutic implication are especially underlined.

keywords: hypohidrose, hypotrichose, hypodontia, dental prosthesis. 